\title{
COMPUTER CONTROL OF THE SPECTRAL COMPOSITION OF THE POWERFUL LASER SYSTEM IRRADIATION WITH A WIDE RANGE OF LASER TRANSITIONS ON METAL VAPORS
}

\author{
Anatoly Soldatov ${ }^{1 \mathrm{a}}$, Bogdan Doroshenko ${ }^{1}$, Ivan Kostadinov ${ }^{2}$, Yury Polunin, Nikola \\ Sabotinov $^{3}$, Alexey Shumeiko ${ }^{1}$ \\ ${ }^{1}$ Tomsk State University, 634050, Tomsk, Russia \\ ${ }^{2}$ Pulslight Ltd., Sofia, Bulgaria \\ ${ }^{3}$ Bulgarian Academy of Sciences, Sofia, Bulgaria
}

\begin{abstract}
The results of the experimental study cycle of the multiwave metal vapor laser system on the basis of the original configuration of the multimedia laser emitter. The spectral parameters of the setup have been controlled using a personal computer (PC). This allows carrying out their independent optimization according to excitation conditions, and, therefore, promptly allocating the output set of oscillating wavelengths and their relative distribution in power, which makes the system attractive for scientific and technological application.
\end{abstract}

\section{Introduction}

Lasers and laser systems are successfully used to solve a lot of applied problems of the atmospheric spectroscopy, in particular remote sensing of atmospheric parameters such as temperature, humidity, concentration, and particle size of various types of aerosol, including man-made products $[1,2]$. Among the latter, metal vapor lasers (MVL) are well represented [3, 4]. Currently, the most effective among MVLs are copper vapor lasers [4, 7] and strontium vapor lasers [5, 6], operating with pulse repetition rates from units of hertz up to hundreds of kilohertz. The work [1] describes an oscillator operating on vapors of $\mathrm{CuBr}$, Srli, and SRII, when two active elements (AE) are placed into a common resonator. The paper presents the design of a laser system in which the system "oscillator-amplifier" is used to increase the average power and the energy in the lasing pulse. Active environments on $\mathrm{CuBr}$ and $\mathrm{Sr}$ vapors are used in $\mathrm{AE}$. The proposed device allows operating in a multiwave laser system controlled via a personal computer (PC), as the adjustment of the delay time of lasing pulses between the elements of the master oscillator and the amplifier, consisting of $\mathrm{AE}$ with different environments, is lightened with the use of autonomous high-voltage power sources. The control and synchronization unit (CSU) is directly connected to the PC.

\footnotetext{
${ }^{\text {a }}$ Corresponding author: general@tic.tsu.ru
} 


\section{Experimental setup}

Figure 1 represents a schematic diagram of the experimental setup. The basis of the setup were active laser elements on $\mathrm{CuBr}$ and $\mathrm{Sr}$ vapors that were united into the classic scheme "oscillator - amplifier". The master oscillator was composed of gas discharge tubes (1) with the active zone $L=800 \mathrm{~mm}, D=26 \mathrm{~mm}$, and a gas discharge tube (2) with the active zone $L=550 \mathrm{~mm}, D=30 \mathrm{~mm}$. They were located in the common resonator consisting of spherical mirrors: convex (3) and concave (4). The collimator was comprised of two concave spherical mirrors $(5,6)$ coated with aluminum and placed into the common focus of spatial filter mirrors (7) with a hole diameter of $0.3 \mathrm{~mm}$, which has allowed to allocate from the oscillator irradiation a spatial component with a spreading close to the diffraction limit. GDT of the amplifier (7) with the active zone $L=1000 \mathrm{~mm}$ and a diameter $D=35 \mathrm{~mm}$, and (8) $L=550 \mathrm{~mm}$ and a diameter $D=30 \mathrm{~mm}$. have large (as compared to GDT of the generator) diameters of the active channel. Excitation pulses were fed to GDT electrodes from high-voltage power sources $(10-13)$ executed on the basis of converters on powerful transistors with a pulse charge of operating capacitances and hydrogen thyratrons TGI-1 $1000 / 25$ as switches, followed by a two-stage compression carried out by ferromagnetic compression cells. The control and synchronization unit (29) consisted of the master oscillator $(29 a)$, the delay module $(29 b)$, and the synchronization module $(29 c)$, used for adjusting the relative delay time of the pumping pulse between each active element of the laser system and allowing to change the lasing pulse repetition rate over a wide range. This mode provides stable energy in the lasing pulse, since the pumping parameters and the thermal mode of the active media remained unchanged. The synchronization module had a connector for an external source of computer control (30). The laser performance measurement system allowed controlling spectral and power characteristics. Measurement of current-voltage values, such as the amplitude and the duration of high-voltage pumping pulse (voltage on GDT and the current through the tube), with the use of voltage dividers $(14,16,18,20)$ and low-resistance shunts $(15,17,19,21)$, was carried out to optimize excitation conditions and coordinate active media. Excitation pulses were recorded using the "Tektronix" oscilloscope. The emission spectra were measured with the use of a spectral filter unit (23) a monochromator MSDD-1000 (24), according to changes in parameters of active media coordination. A power meter "OPHIR" was used to control the output power of the laser. Using a PC (30) it is possible to specify commands to the control and synchronization unit (CSU), with a feedback from the controller (29), thereby to allocate a region of the spectrum necessary for a particular application from the entire irradiation range. Since the excitation of each AE is carried out with the use of its own high-voltage power source, there is a possibility to carry out an independent optimization of pumping conditions for any selected active medium. It should be noted that a very high gain in active media allows obtaining generation on a large transition of near and infrared media without anti-reflecting coatings on windows. 


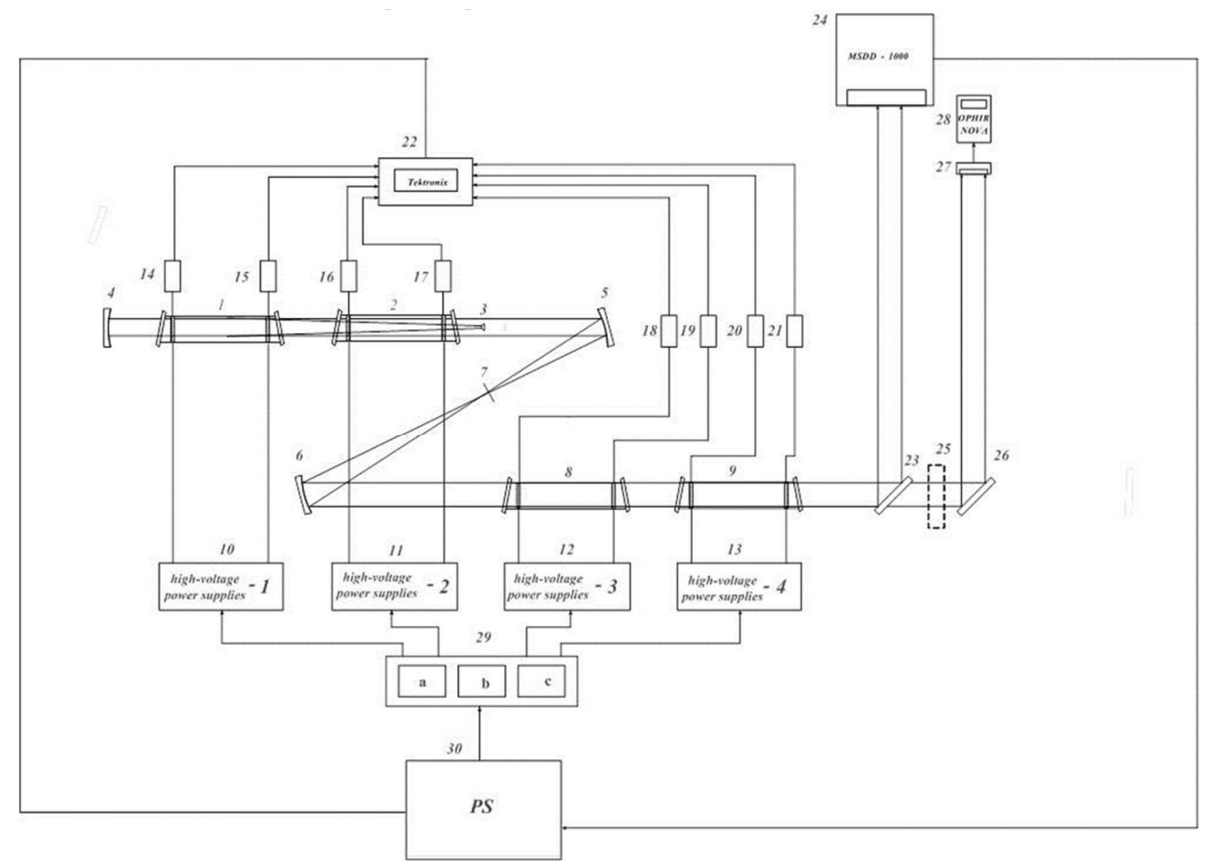

Figure 1. The schematic of the experimental setup:

1 - GDT-1, CuBr; 2 - GDT-2, Sr; 3 - convex mirror; 4 - curved mirror; 5,6 - concave spherical mirrors; 7 - spatial filter; 8 - GDT-3, CuBr; 9 - GDT-4, Sr; 10,11,12,13 - high-voltage power supplies, 14,16,18,20 - voltage dividers; 15, 17,19,21 - low-resistance shunts; 22 - multi-channel oscilloscope "Tektronix"; 23, 26, 30 - rotary mirror; 24 - monochromator MSDD-1000; 25 - unit of spectral filters, 27 - indicator "OPHIR-NOVA"; 28 - gauge "OPHIR-NOVA"; 29 - control and synchronization unit (CSU); 30 - PC.

\section{Results and their discussion}

The studies on the generation at wavelengths of vapors of the atom $\mathrm{CuI}(0.51,0.58)$, ion SrII $(1.03,1.09)$ and atom SrI $(2.60,2.69,2.92,3.01,3.06$ and $6.45 \mu \mathrm{m})$ have been carried out using the setup. Table 1 shows the average power of laser transitions, the average lasing power in individual laser transitions, depending on the delay time of excitation pulses of active elements on $\mathrm{CuBr}$ vapors $\left(\tau_{1}\right)$ and $\mathrm{Sr}$ vapors $\left(\tau_{2}\right)$.

Table 1. Average powers of laser transitions $(\mathrm{Sr}+\mathrm{CuBr})$.

\begin{tabular}{|c|c|c|c|c|c|c|c|c|}
\hline No & $\begin{array}{c}P_{\text {ave }}, W \\
(0,51 \\
\mu \mathrm{m})\end{array}$ & $\begin{array}{c}\mathrm{P}_{\text {ave }}, \mathrm{W} \\
(0,578 \\
\mu \mathrm{m})\end{array}$ & $\begin{array}{l}\mathrm{P}_{\mathrm{ave}}, \mathrm{W} \\
(1.03 ; 1.09 \\
\mu \mathrm{m})\end{array}$ & $\begin{array}{l}P_{\text {ave }}, W \\
(2.6-3.06 \\
\mu \mathrm{m})\end{array}$ & $\begin{array}{c}\mathrm{P}_{\text {ave }}, \mathrm{W} \\
(6.45 \\
\mu \mathrm{m})\end{array}$ & \multirow[t]{2}{*}{$\begin{array}{c}\mathrm{P}_{\text {ave }}, \mathrm{W} \\
\text { (total) }\end{array}$} & \multirow[t]{2}{*}{$\begin{array}{l}\tau_{1}, \\
\text { ns }\end{array}$} & \multirow[t]{2}{*}{$\tau_{2}, \mathrm{~ns}$} \\
\hline & \multicolumn{2}{|c|}{$\mathrm{CuBr}$} & \multicolumn{3}{|c|}{$\mathrm{Sr}$} & & & \\
\hline 1 & 14 & 8 & 6 & 9 & 12 & 49 & 20 & 15 \\
\hline 2 & 0 & 0 & 6 & 9 & 12 & 27 & -100 & 15 \\
\hline 3 & 14 & 8 & 0 & 0 & 0 & 22 & 20 & -100 \\
\hline 4 & 0 & 0 & 0.5 & 1 & 8 & 9,5 & -100 & 30 \\
\hline 5 & 3 & 1 & 3 & 5 & 2.5 & 14.5 & 10 & 0 \\
\hline 6 & 2 & 0 & 0.3 & 1 & 8 & 11.3 & 0 & 40 \\
\hline $7 *$ & 0 & 0 & 0.1 & 12 & 1 & 13.1 & 0 & 15 \\
\hline
\end{tabular}


The first line of the table presents data in the optimal synchronization mode for two media; this corresponds to one of the states of the computer program. Each of the following lines represents the altered state. The table lists the intermediate values of most explicit changes in the spectral composition of the irradiation. The interelement system filtration mode is shown in the line $7^{*}$ when the predominant spectral emission range of 2.6 and 3.1 $\mu \mathrm{m}$.

It is known that the active medium on $\mathrm{Cu}$ and $\mathrm{Sr}$ vapor are optically transparent interaction. In our case, within the working channel of active element on $\mathrm{CuBr}$ vapors present a number of compounds $\left(\mathrm{CuBr}, \mathrm{Br}_{2}\right.$, etc.), Having a certain structure of molecular absorption bands, which could lead to an increase in the intracavity losses for lasing lines at crossings strontium. Obtained in the third cycle of measurements experimental data, these fears are largely removed. Figure 2 shows the relative positions of the current pulse through the active element $(\mathrm{CuBr})$ and the pulse of the laser of a copper bromide.

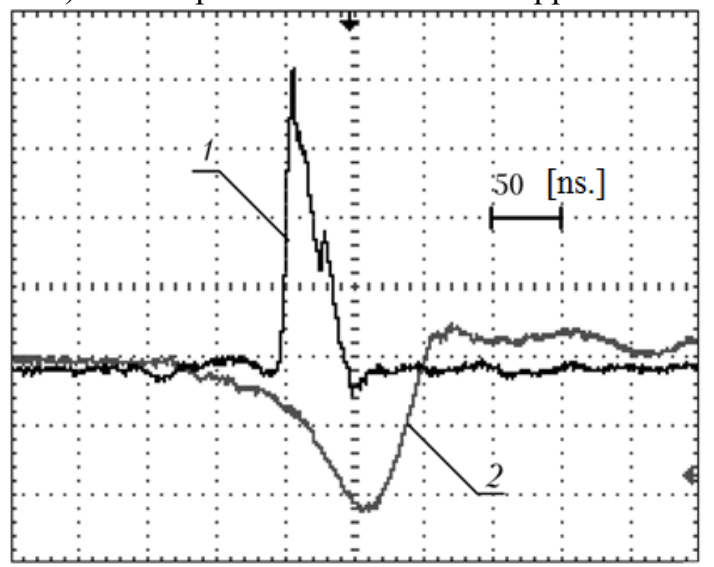

Figure 2. Waveforms generating pulses (1) and a current pulse through $\mathrm{AECuBr}$ vapors to (2).

Figure 2 shows the relative positions of the current pulse through the active element $(\mathrm{CuBr})$ and the pulse of the laser of a copper bromide. It can be seen that the latter is located on the front edge of the current pulse, and the duration of the base generation is $40 \mathrm{~ns}$, which is typical for copper vapor lasers and its compounds in pulse repetition rate within $10 \ldots 20 \mathrm{kHz}$. Waveforms pulse generation on SrI transitions are shown in Figure 3. Pulse generation duration on lines of strontium ion $\lambda \sim 1 \mu \mathrm{m}$ is $50 \mathrm{~ns}$ and is close to the pulse width generating $\mathrm{CuBr}$-laser. The pulse duration on the most powerful line of strontium generation $(\lambda=6.45 \mu \mathrm{m})$ is about $130 \mathrm{~ns}$, the duration of the total generation in the $\mathrm{m}-\mathrm{m}$ transitions $\mathrm{m}-\mathrm{m}$ strontium is $\sim 70 \mathrm{~ns}$. Generation parameters on the transitions of the atom and strontium ion in multielement laser differs little from generation to oneelement Sr-laser. 


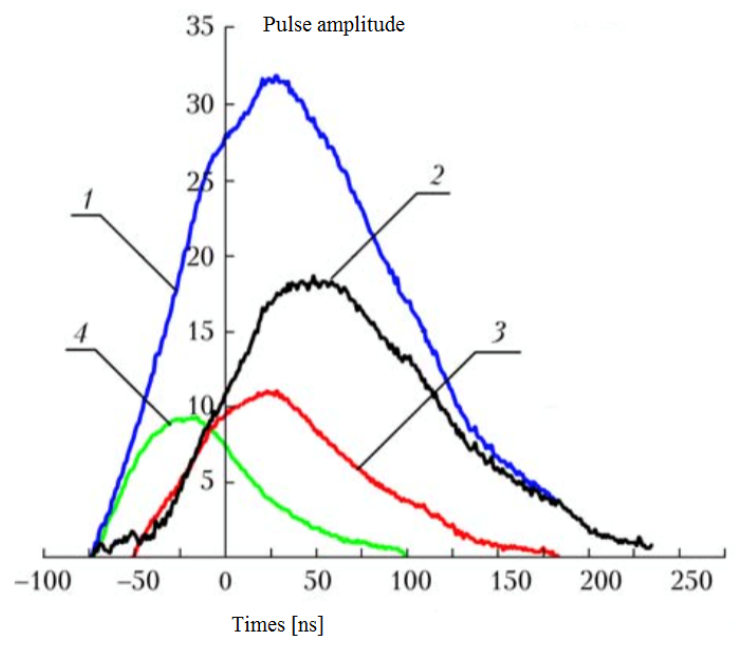

Figure 3. Waveforms of the laser pulses to strontium pairs: 1 - total generating momentum; 2 - impulse generation $\lambda=6.45 \mu \mathrm{m} ; 3$ - impulse generation $\sim 3 \mu \mathrm{m} ; 4$ - generating momentum $\sim 1$ $\mu \mathrm{m}$.

\section{Conclusion}

A computer-controlled system has been presented. The concept allowing controlling the construction of multi-wave laser systems has been proposed. The concept lies in the fact that several gas discharge $\mathrm{AE}$ (two or more) are placed into a single (common) resonator. In the proposed device the adjustment of time coordination between elements of the master oscillator and the amplifier consisting of $\mathrm{AE}$ with different media and the allocation of the spectral composition of the radiation is carried out using a PC. The scheme of a laser system wherein the oscillator and the amplifier are composed of four active elements operating on $\mathrm{CuBr}$ and $\mathrm{Sr}$ vapors has been developed. The laser system is capable to operate in a multi-wave mode with a high average oscillating power. The system can operate in multi-wavelength mode with a high average power lasing at 10 spectral lines in the visible and near-infrared range. Such a configuration of the laser system and the system support allow solving applied problems in the area of the atmosphere monitoring: temperature, humidity, concentration, and particle sizes of various kinds of aerosol, including man-made products.

\section{References}

[1] E.V. Browell, T.D. Wilkerson, and T.J. McCrath, Appl. Opt. 22,522 (1983)

[2] A.N. Soldatov, N.V. Sabotinov, E.L. Latush, G.D. Chebotarev, N.K. Vuchkov, N.A. Yudin, Strontium and calcium vapour lasers. Vol. 1 (Prof. Marin Drinov Academic Publishing House, Sofia, 2013)

[3] P.A. Bokhan, P.P. Gugin, D.E. Zakrevskii, M.A Lavrukhin, M.A. Kazaryan, N.A. $\begin{array}{llll}\text { Lyabin, } \quad \text { Quantum } & \text { Electronics }\end{array}$ doi: 10.1070/QE2013v043n08ABEH015050

[4] C.E. Little, Metal Vapour Lasers. Physics Engineering and Applications (Wiley, United States, 1999)

[5] A.N. Soldatov, S.Ju. Mirza, Ju.P. Polunin, A.S. Shumeyko, I.C. Costadinov, Journal of Applied Spectroscopy 81, 1025 (2015) doi: 10.1007/s10812-015-0045-8 
[6] F. Saleheen, C.-H. Won, 2015 IEEE SENSORS - Proceedings, 7370424 (2015) doi: 10.1109/ICSENS.2015.7370424

[7] G.S Evtushenko, Proceedings of SPIE - The International Society for Optical Engineering 9810, 98101F (2015) doi: 10.1117/12.2225586 